\title{
COLOR BOSONIZATION, CHIRAL PARAMETRIZATION OF GLUONIC FIELD and QCD EFFECTIVE ACTION
}

\author{
VICTOR NOVOZHILOV *and YURI NOVOZHILOV ${ }^{\dagger}$ \\ V.Fock Institute of Physics, St.Petersburg State University, \\ Ulyanovskaya str. 1, 198504, St.Petersburg, Russia
}

\begin{abstract}
We develop a color bosonization approach to treatment of QCD gauge field ("gluons") at low energies in order to derive an effective color action of QCD taking into account the quark chiral anomaly in the case of $\mathrm{SU}(2)$ color.. We have found that there exists such a region in the chiral sector of color space, where a gauge field coincides with of chirally rotated vector field, while an induced axial vector field disappears. In this region, the unit color vector of chiral field plays a defining role, and a gauge field is parametrized in terms of chiral parameters, so that no additional degrees of freedom are introduced by the chiral field. A QCD gauge field decomposition in color bosonization is a sum of a chirally rotated gauge field and an induced axial-vector field expressed in terms of gluonic variables. An induced axial-vector field defines the chiral color anomaly and an effective color action of QCD. This action admits existence of a gauge invariant $\mathrm{d}=2$ condensate of induced axial-vector field and mass.
\end{abstract}

Keywords: [QCD effective action, color chiral model]

PACS Nos.: 12.38.Aw,12.39.Fe

*vnovo@vn9711.spb.edu

†yunovo@pobox.spbu.ru 


\section{Introduction}

It is widely believed that the key point to the infrared QCD and confinement is to find important field configurations in the color space. To this end, convenient parametrization of the gauge field is considered as the first task. This task contains several subtle points, because, due to the phenomenon of chiral anomaly [1, the total color space unifies gauge and chiral (anomalous) sectors in the unique sector with topological properties. The gauge sector was extensively studied in pursuite of the monopole condensation scenario for confinement [2]. Recently, color Hopf-type solitons were reported by Faddeev and Niemi [3] on the basis of n-field model of infrared QCD by Faddeev [4]. This result generated a series of papers explaining the place of the n-model in infrared QCD [5], 6], its relation to the Cho decomposition of the QCD gauge field [7, 8], as well as transformation properties [9]. In quest for infrared variables in QCD two versions of parametrization of the gauge field in terms of dual variables were proposed by Faddeev and Niemi [10]. The chiral color sector at low energies was studied less. The color chiral bosonization [11] describes this sector in terms of an effective action, and it was found that such an action with a chromomagnetic vacuum background field admits stable soliton - skyrmion solutions [12].

In the present paper we develop chiral color bosonization approach to low energy QCD in combined gauge and chiral sectors, when quark color chiral degrees of freedom are explicitly taken into account. The aim of the paper is to find the Lagrangian describing the quark color chiral contribution to QCD dynamics.

We assume that bosonization is possible in the color case and introduce local chiral color transformation of quarks, thereby extending quark color group to the Left-Right group and including the chiral color anomaly into consideration. This approach has an advantage of well defined initial point, i.e. two separate color sectors - gluons and chirally rotated quarks - with definite transformation properties (Left-Right color group). We have an initial gauge field $V_{\mu}$ and two fields arising in its chiral transformation $U$, namely, a gauge field $V_{\mu}^{U}$ and an axial vector field $A_{\mu}^{U}$.However, introducing the quark color chiral field $U$ we arrive at a system with too many degrees of freedom. A consistent parametrization of gluons and the chiral field within Left-Right color group becomes a special task. To eliminate superfluous variables, one should consider a relation between gluonic fields $V_{\mu}$ and chirally transformed field $V_{\mu}^{U}$ and find how different parts of these fields can be made from the same material, so that chiral field variables are either fixed, or fully incorporated into gauge field variables. This is a key point in our color bosonization approach. When this task is accomplished, one can integrate chiral color anomaly and get an effective action, as a sum of bosonization action and the Yang-Mills action. We hope that this effective action will be helpful in low energy QCD.

We consider gluons and quarks at one-loop level in the background gauge. Then gauge fields $V_{\mu}, V_{\mu}^{U}$ and an axial vector $A_{\mu}^{U}$ in the Dirac Lagrangian are background ones. One-loop quark chiral terms represent bosonization corrections to the Yang-Mills 
action for the background gauge fields. One-loop gluon ic action is of no interest for the present paper.

In order to find, how vectors $\left(V^{U}, A^{U}\right)$ are expressed in terms of gluonic field $V_{\mu}$ variables, we look at first for a region $\Omega$ in color space and such a gauge field there $V_{\mu} \equiv V_{\mu}^{\Omega}$, which is invariant under chiral transformation: $\left(V_{\mu}^{\Omega}\right)^{U}=V_{\mu}^{\Omega}$. Such a gauge field $V_{\mu}^{\Omega}$ will contain $U$-variables. With $V_{\mu}=V_{\mu}^{\Omega}$, an axial vector field should be absent $A_{\mu}^{\Omega}=0$ and quark color chiral anomaly disappear. This case provides us with a partial parametrization of gluonic field in terms of chiral parameters. In order to investigate this point in the classical theory with background fields we need a relation expressing gluonic field $V_{\mu}$ in terms of chirally dependent field $V_{\mu}^{U}(x)$, or the determinant $\operatorname{det}\left(\partial V / \partial V^{U}\right)$. In quantum theory we need to know how changes the measure $d \mu_{V} \rightarrow d \mu_{V}^{U}$ of the gluonic path integral because of replacement $V_{\mu}(x) \rightarrow V_{\mu}^{U}(x)$.

We develop the theory at example of the color $\mathrm{SU}(2)$ group. An extension to the color $\mathrm{SU}(3)$ will use many points of the $\mathrm{SU}(2)$ case [15].

\section{Bosonization in the flavor case}

In this section we review the bosonization approach to the effective chiral action in the case of chiral flavor. We consider massless fermions in external vector and axial vector fields $V_{\mu}, A_{\mu}$. The path integral of fermions $Z_{\psi}[V, A]$ is a functional of $V, A$ :

$$
Z_{\psi}[V, A]=\int d \mu_{\psi} \exp i \int d x \bar{\psi} D(V, A) \psi
$$

where $\not D=i \gamma^{\mu}\left(\partial_{\mu}+V_{\mu}+i \gamma_{5} A_{\mu}\right)$ is the Dirac operator. The chiral transformation of fermions is given by

$$
\psi_{L}^{\prime}=\xi_{L} \psi_{L}, \psi_{R}^{\prime}=\xi_{R} \psi_{R}, \psi=\psi_{L}+\psi_{R}
$$

where $\xi_{L}(x)$ and $\xi_{R}(x)$ are local chiral phase factors of left and right quarks $\psi_{L}$ and $\psi_{R}$, represented by unitary matrices in defining representations of left $S U(N)_{L}$ and right $S U(N)_{R}$ subgroups of the chiral group $G_{L R}=S U(N)_{L} \times S U(N)_{R}$. For $\psi_{L}=\frac{1}{2}\left(1+\gamma_{5}\right) \psi, \psi_{R}=\frac{1}{2}\left(1-\gamma_{5}\right) \psi$, generators $t_{L a}$ and $t_{R a}$ of left and right subgroups

of $G_{L R}$ can be written as $t_{L a}=\frac{1}{4}\left(1+\gamma_{5}\right) \tau_{a}, t_{R a}=\frac{1}{4}\left(1-\gamma_{5}\right) \tau_{a},\left[t_{L a}, t_{R b}\right]=0$, where $\tau_{a}, a=1,2,3$ are the Pauli matrices. Then quark left and right chiral phase factors $\xi_{L}, \xi_{R}$ arise from application of operators $\hat{\xi}_{L}=\exp \left(-i t_{L a} \omega_{L a}\right), \hat{\xi}_{R}=\exp \left(-i t_{R a} \omega_{R a}\right)$ to left and right quarks $\psi_{L}$ and $\psi_{R}$. Vector gauge transformations $g(x)$ are associated with $t_{a}=t_{L a}+t_{R a}=\tau_{a} / 2$, i.e. $g(x)$ has properties of the product $\hat{\xi}_{L}(x) \hat{\xi}_{R}(x)$ of identical left and right rotations, $\omega_{L}=\omega_{R}=\alpha$. The generator of purely chiral transformations $g_{5}(x)$ is $t_{5 a}=\gamma_{5} \tau_{a} / 2=t_{L a}-t_{R a}$; thus, $g_{5}(x)$ has properties of $\hat{\xi}_{L}(x) \hat{\xi}_{R}^{+}(x)$ for $\omega_{L}=\omega_{R}=\Theta$. Infinitesimally, the Dirac operator is transformed according to

$$
\delta \not D=\left[i \frac{1}{2} \alpha_{a} \tau_{a}, D\right]+\left\{i \frac{1}{2} \gamma_{5} \Theta_{a} \tau_{a}, \not D\right\}
$$


Commutation relations for $t_{a}, t_{5 a}$ are given by

$$
\left[t_{a}, t_{b}\right]=i \varepsilon_{a b c} t_{c},\left[t_{a}, t_{5 b}\right]=i \varepsilon_{a b c} t_{5 c},\left[t_{5 a}, t_{5 b}\right]=i \varepsilon_{a b c} t_{c}
$$

Instead of phases $\xi_{L}$ and $\xi_{R}$ one can work with the chiral field $U=\xi_{R}^{+} \xi_{L}$, which describes rotation of only left quark leaving right quark in peace $\psi_{L} \rightarrow \psi_{L}^{\prime}=$ $U \psi_{L}, \psi \rightarrow \psi_{R}^{\prime}=\psi_{R}$. The same result can be obtained by the chiral transformation $\psi_{L} \rightarrow \xi_{L} \psi_{L}, \psi_{R} \rightarrow \xi_{R} \psi_{R}$, followed by a vector gauge transformation with a gauge function $\xi_{R}^{+}$. The usual chiral gauge choice is $\xi_{R}=\xi_{L}^{+}$, then the chiral field is taken as squared left chiral phase: $U=\xi_{L}^{2}$.

The chiral tranformation of fermions in the Dirac action is equivalent to the following change of the Dirac operator

$$
\begin{gathered}
\bar{\psi}^{\prime} D(V, A) \psi^{\prime}=\bar{\psi} D\left(V^{U}, A^{U}\right) \psi \\
V^{U}=\frac{1}{2}\left[U^{+}(\partial+V+A) U+(V-A)\right] \\
A^{U}=\frac{1}{2}\left[U^{+}(\partial+V+A) U-(V-A)\right]
\end{gathered}
$$

A transformed fermionic path integral $Z_{\psi}^{\prime}$ because of $\psi \rightarrow \psi^{\prime}$ in the Dirac action is equal to an original path integral as a functional of transformed fields $Z_{\psi}^{\prime}[V, A]=$ $Z_{\psi}\left[V^{\prime}, A^{\prime}\right] . Z_{\psi}$ is invariant under vector gauge transformations of fermions, but undergoes changes under chiral tranformations, because of non-invariance of the fermionic measure $d \bar{\psi} d \psi[13$ : chiral transformations are anomalous. The chiral anomaly $\mathcal{A}$ is defined by an infinitesimal change of $\ln Z_{\psi}$ due to an infinitesimal chiral transformation $\delta g_{5}=i \theta_{a} \tau_{a} \equiv \Theta$.

We put $g_{5}(s)=\exp \gamma_{5} \Theta s$ and write the anomaly $\mathcal{A}(x, \Theta)$ at a chiral angle $\Theta$

$$
\mathcal{A}(x, \Theta)=\frac{1}{i}{\frac{\delta \ln Z_{\psi}(\exp \Theta s)}{\delta s}}_{s=1}
$$

The usual way [14] to calculate effective chiral action $W_{\text {eff }}$ is to find the anomaly and integrate it over $s$ up to $g_{5}=\exp \gamma_{5} \Theta$.

$$
W_{e f f}=-\int d^{4} x \int_{0}^{1} d s A(x ; s \Theta) \Theta(x)=\int d^{4} x L_{e f f}-W_{W Z W}
$$

where the Wess-Zumino-Witten term $W_{W Z W}$ describes topological properties of $g_{5}$ (and $U$ ) and is represented by a five-dimensional integral with $x_{5}=s$. It is the analogue of $W_{\text {eff }}$ for color that we are interested in.

\section{Color bosonization}

The basic color fields are the Yang-Mills field $V_{\mu}(x)$ and the quark field $\bar{\psi}(x), \psi(x)$ with the Lagrangian 


$$
L_{\psi}=\bar{\psi} \not D(V) \psi
$$

where $D(V)$ is the Dirac operator for massless quarks with the Yang-Mills field $V_{\mu}$. There are no dynamical axial vector field: $A_{\mu}=0$.

We consider the vacuum functional $Z$ for the system quarks + gluons as being always in the presence of the color chiral field $U(x)$ describing local chiral degrees of freedom of quarks $\bar{\psi}, \psi$ and resulting in replacement of $Z_{\psi}[V]$ by $Z_{\psi}\left[V^{U}, A^{U}\right]$, where $V^{U}$ and $A^{U}$ are vector and axial vector fields arising in the Dirac operator $/ D(V) \rightarrow \not D\left(V^{U}, A^{U}\right)$ from the gluonic field $V_{\mu}$ due to chiral rotation

$$
Z=\int d \mu_{V}\left\{\exp i \int d x L_{Y M}(V)\right\} Z_{\psi}\left[V^{U}, A^{U}\right]
$$

The vacuum functional $Z$ depends on color degrees of freedom of quarks and gluons. The gluon measure $d \mu_{V}$ includes only vector color degrees, while the quark functional $Z_{\psi}$ contains both vector and chiral color degrees in the quark measure $d \bar{\psi} d \psi$. Explicitly $\mathrm{Z}_{\psi}$ depends only on gluonic field $V_{\lambda}$. Under transformations of the color gauge group $S U(2)_{c}=S U(2)_{L+R}$ the vacuum functional is invariant, $\delta \mathrm{Z}=0$. The existence of chiral anomaly means that $\delta Z \neq 0$ under chiral transformations belonging to the coset $G_{L R} / S U(2)_{L+R}$, because of non-invariance of the measure [13].

While in the flavor case $V_{\mu}$ and $U$ are always independent variables, in the color case two types of questions are posiible:

(a) what is an action for the chiral field in a given gluon field. For example, what is an action for chiral soliton in color vacuum field [11. Total number of variables should not exceed that of dynamical gluon field. This question is of the same type as in the flavor case, and an action is given by an expression (7) for flavor one.

(b) What is an anomalous action for color variables taking into account that a pair $\left(V^{U}, A^{U}\right)$ should contain the same number of variables as $V$. Then an initial path integral is $Z_{U}$ in (9) with all color variables $\left(V^{U}, A^{U}\right)$ of left-right group shown explicitly. In this case, an anomalous action is defined by the expression, which formally is of opposite sign compared with the flavor case (7). The "bosonized", or anomalous action is defined by the expression

$$
W_{\text {bose }}\left[V^{U}, A^{U}\right]=-i\left(\ln Z_{\psi}\left[V^{U}, A^{U}\right]-\ln Z_{\psi}[V, 0]\right),
$$

which includes in general two different actions: a topological $W_{W Z W}$ and a nontopological $W_{a n}$ ones. For $\mathrm{SU}(2) W_{W Z W}=0$. For $\mathrm{SU}(3)$ it is the most interesting part.

We consider one loop approximation for gluons in the background gauge in absence of external vector fields. Then $V_{\mu}$ will be a classical (background) field for gluons. There is no background axial vector field $A_{\mu}$. After quark color chiral transformation with the chiral field $U$, we get from $V_{\mu}$ a vector matrix $W_{\mu}^{U}$ containing both $V_{\mu}^{U}$ and $A_{\mu}^{U}$ 


$$
\begin{gathered}
W_{\mu}^{U}=V_{\mu a}^{U} t_{a}+A_{\mu a}^{U} t_{5 a} V_{\mu}^{U}=\frac{1}{2}\left(U^{+} V_{\mu} U+V_{\mu}+U^{+} \partial_{\mu} U\right), \\
A_{\mu}^{U}=\frac{1}{2} U^{+} D_{\mu} U,
\end{gathered}
$$

where $D_{\mu}$ is defined with the background field $V_{\mu}$. When $U=1$ we return to the case, when $A^{U}=0$ and $W_{\mu}^{U}=V_{\mu}$. Note that a chirally rotated gauge field is just an extension of an initial gauge field by an induced axial vector field: $V_{\mu}^{U}=V_{\mu}+A_{\mu}^{U}$.

Thus, in the color bosonization approach, there are three vectors $V_{\mu}, V_{\nu}^{U}, A_{\lambda}^{U}$ living in the common color space of gluons and chiral color of quarks. They include two gauge fields $V_{\mu}$ and $V_{\mu}^{U}$, belonging to different vector-type subgroups of left-right chiral group. $V_{\mu}$ transforms with $L_{a}+R_{a}$ color generators, while $V_{\mu}^{U}$ transforms with generators $L_{a}^{U}+R_{a}$, where the left generator is additionally rotated. We remind that the chiral field $U$ belongs to the anomalous channel $\Theta$.

Because of chiral transformations, asymptopic constraints imposed on gluonic field $V_{\mu}$ lead to constraints for $V_{\mu}^{U}, A_{\mu}^{U}$. It is usually required for the dynamical $V_{\mu}$ that

$$
\int d^{3} x \operatorname{tr} V_{\mu} V_{\mu} \prec \infty
$$

We assume that this property is preserved by chiral transformations. In view of orthogonality of $t_{5 a}$ and $t_{b}$, we have

$$
\int d^{3} x\left\{\operatorname{tr} A_{\mu}^{U} A_{\mu}^{U}\right\} \prec \infty
$$

It means that asymptotically

$$
U^{+} D_{\mu} U \rightarrow 0, r \rightarrow \infty
$$

Consider the chiral field $U=\xi_{L}^{2}$, where $\xi_{L}$ is an $S U(2)_{L}$ rotation in the fundamental representation with generators $\tau_{a} / 2$

$$
\begin{gathered}
\xi_{L}(x)=\exp (i \hat{n} F / 2), 0 \leq F \leq 2 \pi \\
U=\exp 2(i \hat{n} F / 2), \hat{n}=n_{a} \tau_{a}, n_{a} n_{a}=1 \\
U^{+} D_{\mu} U=i \hat{n} \partial_{\mu} F+i \frac{1}{2} D_{\mu} \hat{n} \sin 2 F+\left[\hat{n}, D_{\mu} \hat{n}\right] \frac{1}{2} \sin ^{2} F
\end{gathered}
$$

Then asymptotically at $r \rightarrow \infty$

$$
\partial_{\mu} F \rightarrow 0, D_{\mu} \hat{n} \rightarrow 0
$$

Within the chiral left-right group gauge fields $V_{\mu}$ and $V_{\mu}^{U}$ are associated with different $\mathrm{SU}(2)$ - subgroups. One may conjecture that there is a finite region in the total color space, where both fields are equivalent. Such a region should correspond to restricted 
number of color degrees of freedom. A boundary of this region, where number of variables is changed, will be reflected in behaviour of the determinant $\operatorname{det}[1+R(U)]$.

Consider a change of fields $A_{\mu}(x) \rightarrow A_{\mu}^{\prime}(x)=\frac{1}{2}\left(U^{+}(x) A_{\mu}(x) U(x)+A_{\mu}(x)\right)$, or

$$
A_{\mu a}^{\prime}=\frac{1}{2}\left(\delta_{a b}+R_{a b}(U)\right) A_{\mu b}, R_{a b}(U)=\frac{1}{2} \operatorname{tr}\left(\lambda_{a} U^{+} \lambda_{b} U\right)
$$

where $R_{a b}(U)$ is the transformation $U$ in adjoint representation. We get $3 \times 3$ matrix $R(U)=R\left(\xi_{L}^{2}\right)$ by replacing $S U(2)$ generators $\tau_{a} / 2$ with $S O(3)$ hermitian generators $\mathrm{O}_{a}$

$$
R(U)=\exp i O_{a} n_{a} 2 F=1+i \hat{N} \sin 2 F+\hat{N}^{2}(\cos 2 F-1)
$$

The $3 \times 3$ matrix $\hat{N}=O_{a} n_{a}$ has a property $\hat{N}^{3}=\hat{N}$, so that eigenvalues of $\hat{N}$ are equal to $+1,-1,0$. It follows that $\operatorname{det} \frac{1}{2}(1+R(U))=\frac{1}{2}(1+\cos 2 F)$. Thus, we have singularities at points $x_{s}$, where $F\left(x_{s}\right)=\pi / 2, U_{s} \equiv U\left(x_{s}\right)=i \hat{n}\left(x_{s}\right)$ and $R\left(U_{s}\right)=$ $1-2\left\{\hat{N}\left(x_{s}\right)\right\}^{2}$. At singularity two eigenvalues of the matrix $R(U)$ coincide. In the chiral color space, these singular points $x_{s}$ constitute a spherical surface of radius $F\left(x_{s}\right)$ in the anomalous channel (i.e. which is gauge equivalent to a region of $\gamma_{5} \theta$-parameter of the left-right group), where a general gluonic field $V_{\mu}$ cannot be expressed in terms of chirally dependent field $V_{\mu}^{U}$. The transformation $V_{\mu}[x] \rightarrow \frac{1}{2}[1+R(U)] V_{\mu}(x)$ may be induced by a global chiral rotation, $\partial_{\mu} U=0$; thus, already a global chiral rotation leads to a singular determinant.

Explicitly, using an expression $V_{\mu}^{U}=V_{\mu}+A_{\mu}^{U}$ we get in terms of color vectors $\vec{n}, \vec{V}_{\mu}$

$$
\begin{gathered}
\vec{V}_{\mu}^{U}=\vec{V}_{\mu} \cos 2 F-\frac{1}{2}\left[\vec{V}_{\mu}, \vec{n}\right] \sin 2 F-\vec{n}\left(\vec{V}_{\mu}, \vec{n}\right) \sin ^{2} F+ \\
i \vec{n} \partial_{\mu} F-\frac{1}{2} \partial_{\mu} \vec{n} \sin 2 F-\left[\vec{n}, \partial_{\mu} \vec{n}\right] \sin ^{2} F
\end{gathered}
$$

so that at $F=\pi / 2$ the field $V_{\mu}^{U}$ looses structures represented by $\partial_{\mu} \vec{n}$ and $\left[\vec{V}_{\mu}, \vec{n}\right]$ :

$$
V_{\mu}^{U}=\frac{1}{2}\left(\hat{n} \partial_{\mu} \hat{n}+\hat{n} \hat{V}_{\mu} \hat{n}+V_{\mu}\right), F=\frac{\pi}{2}
$$

while the matrix $\Delta=\frac{1}{2}(1+R(U))$ reduces to $\Delta^{0}=1-\hat{N}^{2}$ with matrix elements $\Delta_{a b}^{0}=n_{a} n_{b}$, and determinant det $\Delta^{0}=0$. The matrix $1-N^{2}$ is a projector on eigenvalue $\hat{N}^{\prime}=0$. Thus, at $F=\pi / 2$, only fields with $N^{\prime}=0$ are essential for construction of singular free connections in color space including chiral degrees of freedom introduced by $U$. In fact, this conclusion follows directly from properties of $\Delta$

$$
(\Delta)_{a b} n_{b}=n_{a},\left(\Delta^{-1}\right)_{a b} n_{b}=(1-i \hat{N} \tan F)_{a b} n_{b}=n_{a}
$$


It reflects (by construction of the chiral field $U=\exp i \hat{n} F$ ) the fact that $U$ commutes locally with a function of $\hat{n}$. In the case, when a relation $U V_{\mu} U^{+}=V_{\mu}$ helds locally, we have det $\Delta=1$.

Let us demonstrate, that it is possible to find such a gluon field $V_{\mu}$ and such a chiral field $U$, that we have an invariance relation $V_{\mu}=V_{\mu}^{U}$. We represent $V_{\mu}$ in the form $V_{\mu}=C_{\mu} \hat{n}+\frac{1}{2} U \partial_{\mu} U^{+}$, where $C_{\mu}$ is an abelian gauge field and check the relation $V_{\mu}^{U}=\frac{1}{2}\left(U V_{\mu} U^{+}+V_{\mu}+U \partial_{\mu} U^{+}\right)=V_{\mu}$. It can be satisfied if $U=i \hat{n}$ or $F=\pi / 2$. We denote this special field on the sphere $\Omega(\pi / 2)$ by $V_{\mu}^{\Omega}$

$$
\begin{gathered}
V_{\mu}^{\Omega}=C_{\mu} \hat{n}+\frac{1}{2} \hat{n} \partial_{\mu} \hat{n} \\
A_{\mu}^{\Omega}=\frac{1}{2}\left(U V_{\mu}^{\Omega} U^{+}-V_{\mu}+U \partial_{\mu} U^{+}\right)=0
\end{gathered}
$$

The chiral field $U$ and the gauge field $V_{\mu}$ contain now the same unit color vector $\hat{n}$. Second term in $V_{\mu}^{\Omega}$ satisfies separately the equivalence relation $V_{\mu}^{U}=V_{\mu}$. The field $V_{\mu}^{\Omega}$ is a basic field in the color bosonization approach, because then an axial vector $A_{\mu}^{U}(\Omega)=0$; both the Yang-Mills action $I_{Y M}\left(V^{\Omega}\right)$ and the quark integral $Z_{\psi}\left[V^{U}, A^{U}\right]$, which is in $\Omega$ just $Z_{\psi}\left[V^{\Omega}, 0\right]$, depend on the same $V^{\Omega}$ only. There is no color chiral anomaly -neither a topological one (for $\mathrm{SU}(3)$ ), nor of a non-topological type. The field $V_{\mu}^{\Omega}$ can be obtained by chiral transformation from the simplest vector field, namely, from an abelian field $V_{\mu}^{0}=C_{\mu} \hat{n}$. Also, $V_{\mu}^{\Omega}$ is invariant under the gauge transformation with the chiral field $U=i \hat{n}$ and under chiral transformation

$$
V_{\mu}^{\Omega}=\left(V_{\mu}^{0}\right)^{U}=\frac{1}{2}\left(\hat{n} V_{\mu}^{0} \hat{n}+V_{\mu}^{0}+\hat{n} \partial \hat{n}\right), V_{\mu}^{\Omega}=\hat{n} V_{\mu}^{\Omega} \hat{n}+\hat{n} \partial_{\mu} \hat{n}
$$

The corresponding Yang-Mills field strength $V_{\mu \nu}^{\Omega}$ is

$$
V_{\mu \nu}^{\Omega}=C_{\mu \nu} \hat{n}+\frac{1}{4}\left[\partial_{\mu} \hat{n}, \partial_{\nu} \hat{n}\right]
$$

The field $V_{\mu}^{\Omega}$ depends on four degrees of freedom, instead of required 6 degrees in the case of $\mathrm{SU}(2)$. The field $V_{\mu}^{\Omega}$ was introduced as a starting point of $n$-model [4] (Faddeev) and "Restricted gauge theory" 7](Cho).

Fixing det $\Delta=1$ corresponds to excluding the Cartan mode $\exp i \tau_{3} F$ from the chiral field $U=\exp i \hat{n} F$. In terms of $S U(2)_{L} \times S U(2)_{R}$ generators $\tau_{a} / 2, \gamma_{5} \tau_{b} / 2$, it means that we rotate $\hat{n}$ to $\tau_{3}$ and then fix $\gamma_{5} \tau_{3}-$ parameter $F$.

\section{Gluonic chiral anomaly for basic field $V_{\mu}^{\Omega}$, as a background field, and kinetic term for $n$ - field}

A quantum expression for the Jacobian of $V_{\mu} \rightarrow V_{\mu}^{U}$ transformation is given by the chiral anomaly. 
Consider the path integral $Z[U]$ in the background gauge, where the chiral field $U$ is in region $\Omega$ of the chiral color space, i.e in the space of chiral parameters $\theta \hat{=} \hat{n}$. We have a gluonic background field $V_{\mu}^{\Omega}$ in gluonic part of $Z[U]$ and a background field $V_{\mu}^{U}=V_{\mu}+A_{\mu}^{U}$ in fermionic path integral $Z_{\psi}\left[V_{\mu}^{U}, A_{\mu}^{U}\right]$

$$
Z[U]=\int d \mu_{Q} \exp i L_{e f f}(V+Q) Z_{\psi}\left[V^{U}, A^{U}\right]
$$

where $Q_{\mu}$ is a quantum field and $G_{\mu}=V_{\mu}+Q_{\mu}$ is a dynamical gluon field. In $\Omega$, these background fields are equal, while $A_{\mu}^{U}=0$, so that QCD in $\Omega$ is associated with the path integral

$$
Z^{\Omega}=\int_{\Omega} d \mu_{Q} \exp i L_{e f f}(V(\Omega)+Q) Z_{\psi}[V(\Omega), 0]
$$

extended over color vector variables $Q_{\mu}$. In the case of bosonization, the anomaluos action resulting from integration of non-abelian anomaly of fermionic path integral $Z_{\psi}$ is a function of axial vector fields $A_{\mu}^{U}$. Thus, in $\Omega$ there is no anomalous color action arising from $Z_{\psi}$.

In the complete color space, the anomalous action is composed of two pieces coming from the gluonic part and from bosonization. Let us retain only the gluonic part. Then we should start by considering the path integral $Z_{a n}[U]$ over non-chirally transformed gluons $G_{\mu}$ and ghosts (measure $[d G]$ ) with an effective lagrangian $L_{e f f}\left(G^{U}\right)$ for a chirally transformed field $G^{U}$ and ghosts

$$
Z_{a n}[U]=\int[d G]\left\{\exp i \int d x L_{e f f}\left(G^{U}\right)\right\}
$$

The effective lagrangian $L_{e f f}$ includes the Yang-Mills part $L_{Y M}$, gauge fixing term $L_{G F}$ and ghost lagrangian $L_{F P}$. The ratio $Z_{a n}[U] / Z_{a n}[U=1]$ gives us the Jacobian of $\left(V \rightarrow V^{U}\right)$ - transformation.

We are interested in one-loop part of $L_{e f f}\left(G^{U}\right)$ in the general case for the field $G_{\mu}$ and an arbitrary $U$. We introduce a background field $B_{\mu}(x)$ and write

$$
G_{\mu}=B_{\mu}+Q_{\mu}
$$

where $B_{\mu}$ transforms inhomogeneously

$$
B_{\mu}^{\prime}=g B_{\mu} g^{+}+g \partial_{\mu} g^{+}
$$

under gauge transformations $g(x)$, while $Q_{\mu}^{\prime}=g Q_{\mu} g^{+}$.

Due to linear relation between $Q_{\mu}^{U}$ and $Q_{\mu}$, one-loop part $L_{1 L}(Q)$ of the effective lagrangian $L_{\text {eff }}\left(G^{U}\right)$ in $Q_{\mu}$ follows immediately from one-loop part $L_{1 L}\left(Q^{U}\right)=$ $Q_{\mu}^{U} K_{\mu \nu}\left(B^{U}\right) Q_{\nu}^{U}$ in $Q_{\mu}^{U}$ 


$$
L_{1 L}(Q)=Q \Delta^{T} K\left(B^{U}\right) \Delta Q
$$

where matrix $K_{\mu \nu}^{a b}\left(B^{U}\right)$ depends on rotated background field $B_{\mu}^{U}$, and the matrix $\Delta_{a b}=\frac{1}{2}(1+R(U))_{a b}$ includes a color rotation $R_{a b}=\frac{1}{2} \operatorname{tr}\left(U \tau_{a} U^{+} \tau_{b}\right), \Delta^{T}$ is transposed of $\Delta$. For $K_{\mu \nu}\left(B^{U}\right)=D^{2}\left(B^{U}\right) \delta_{\mu \nu}-2 B_{\mu \nu}^{U}$ we have

$$
\begin{gathered}
D_{\lambda}\left(B^{U}\right)=D_{\lambda}\left(B+A^{U}\right)=D_{\lambda}(B)+\left[A_{\lambda}^{U}, *\right] \\
B_{\mu \nu}^{U}=\left(B+A^{U}\right)_{\mu \nu}= \\
B_{\mu \nu}+D_{\mu}(B) A_{\nu}^{U}-D_{\nu}(B) A_{\mu}^{U}+\left[A_{\mu}^{U}, A_{\nu}^{U}\right]
\end{gathered}
$$

Thus, the one-loop quantum corrections to the Jacobian $J\left(\partial G_{\mu}^{U} / \partial G_{\mu}\right)$ arising from integration over variables $Q_{\mu}$ with background field $B_{\mu}$ are given by

$$
\operatorname{Tr} \ln \left\{\Delta^{T} K\left(B^{U}\right) \Delta\right\}_{\text {Reg }}
$$

where Reg denotes regularization.

The special case of a background field in $\Omega$ corresponds to $B_{\mu}=V_{\mu}=V_{\mu}^{\Omega}, A_{\mu}^{U}=0$ and $\Delta=1-N^{2}$; so that $\Delta$ becomes a projector on $N^{\prime}=0$ state. Then the matrix $\Delta^{T} \Delta=M=1-N^{2}$ is also the projector, while the one-loop expression for gluonic chiral anomalous action reduces to the standard one-loop gluodynamics taken with $\Delta-$ projectors from both sides

$$
\operatorname{Tr} \ln \left\{\Delta^{T}\left[p^{2}+D^{2}+2 p D-2 V\right] \Delta\right\}_{\text {Reg }}+\text { (ghosts) }
$$

However, the term $\ln \Delta^{T} \Delta$ is not defined by such a direct replacement $\Delta \rightarrow 1-N^{2}$. In order to give meaning to expressions like $\ln \Delta$ and be sure that we can drop $\Delta^{\prime}$ s from $\Delta p^{2} \Delta$ term in (29), we need at the first step to retain in $\Delta$ the terms with projector $\mathrm{N}^{2}$ on states with $N^{\prime}= \pm 1$. Then terms quadratic in $\partial_{\mu} N^{2}$ will give us the kinetic lagrangian

$$
-\Lambda^{2} \frac{1}{2} \operatorname{tr} D_{\mu} N^{2} N^{2} D_{\mu} N^{2} \rightarrow \frac{\Lambda^{2}}{2} \partial_{\mu} n_{a} \partial_{\mu} n_{a}
$$

, where $\Lambda$ is a cut-off.

\section{QCD-SU $(2)_{c}$ at low energies: gauge field and the effective gluonic action}

The field $V_{\mu}^{\Omega}$ is a common part of initial gauge field $V_{\mu}$ and chirally rotated version $V_{\mu}^{U}$. In left-right group without dynamical $A_{\mu}$ these gauge fields are interrelated by $V_{\mu}=V_{\mu}^{\Omega}-A_{\mu}^{U}$. Chiral rotation of quarks $i \hat{n}$ transforms $Z \psi\left[V^{\Omega}-A^{U}, 0\right]$ into $Z_{\mu}\left[V^{\Omega}, A^{U}\right]$, acting as a shift operator. $A_{\mu}^{U}$ should anticommute with $\hat{n}$. Denoting $A_{\mu}^{U}=-X_{\mu}$ we come to the decomposition for the QCD gauge field 


$$
V_{\mu}=V_{\mu}^{\Omega}+X_{\mu}
$$

This decomposition for the QCD gauge field was discussed by Cho [7] in different approach.

For color bosonization approach anticommutativity relation $\left\{\hat{n}, X_{\mu}\right\}=0$ is essential. Due to this property of $X_{\mu}$, a chirally rotated gauge field $V_{\mu}^{U}=\left(V_{\mu}^{\Omega}+X_{\mu}\right)^{U}=V_{\mu}^{\Omega}$ is independent of $X_{\mu}$, while the axial field $A_{\mu}^{U}$ picks up the value $A_{\mu}^{U}=-X_{\mu}$. Thus, this expression for a gauge field $V_{\mu}$ is, at the same time, a decomposition of a gauge field into chirally rotated vector part $V_{\mu}^{U}=V_{\mu}^{\Omega}$ and chirally rotated axial vector part $A_{\mu}^{U}=-X_{\mu}$. In this form, it is explicitly seen that the gluon sector and the quark sector are built on the same color variables, and theYang-Mills action $I_{Y M}(V)$ and bosonization part $Z_{\psi}\left[V^{\Omega},-X\right]$ contain the same set of background fields $\left(V_{\mu}^{\Omega}, X_{\mu}\right)$. In the $\mathrm{SU}(2)$ case, an axial field $A_{\mu}^{U}=-X_{\mu}$ leads to a non-topological chiral anomaly of the quark integral $Z_{\psi}$. In the case $\mathrm{SU}(3)$ the chiral anomaly will include also a topological term. Thus, in this decomposition of $V_{\mu}$, the field $X_{\mu}$ is responsible for the chiral anomaly, and consequently, for a bosonization action. Such an action together with the Yang-Mills action and kinetic term will determine low energy color dynamics.

The color bosonization action $W_{a n}$ can be written in analogy with the flavor case [14. In our notations, the non-topological part of $W_{a n}$ corresponds to the following Lagrangian $L_{a n}=L_{+}\left(V_{\mu}^{\Omega},-X_{\mu}\right)-L_{+}\left(V_{\mu}^{\Omega}+X_{\mu}, 0\right)$ in the Minkowski space

$$
\begin{gathered}
L_{a n}=\frac{\Lambda^{2}}{4 \pi^{2}} \operatorname{tr} X_{\mu}^{2}-\frac{1}{12 \pi^{2}} \operatorname{tr}\left\{\frac{1}{4}\left(V_{\mu \nu}^{\Omega}\right)^{2}+X_{\mu} V_{\mu \nu}^{\Omega} X_{\nu}-\right. \\
\left.\frac{1}{2}\left[D_{\mu}^{\Omega}, X_{\mu}\right]^{2}-\frac{1}{4}\left[X_{\mu}, X_{\nu}\right]^{2}+\left(X_{\mu}^{2}\right)^{2}\right\}+\frac{1}{48 \pi^{2}} \operatorname{tr}\left(V^{\Omega}+X\right)_{\mu \nu}^{2}
\end{gathered}
$$

where $D^{\Omega}$ contains the field $V_{\mu}^{\Omega}$, while

$$
V_{\mu \nu}^{\Omega}=C_{\mu \nu} \hat{n}+\frac{1}{4}\left[\partial_{\mu} \hat{n}, \partial_{\nu} \hat{n}\right]
$$

is the field strength of $V_{\mu}^{\Omega}$.

The Yang-Mills Lagrangian for $V_{\mu}=V_{\mu}^{\Omega}+X_{\mu}$ is given by

$$
L_{Y M}=L_{Y M}^{\Omega}+\frac{1}{2 g^{2}} \operatorname{tr}\left\{\left(D_{\mu}^{\Omega} X_{\nu}-D_{\nu}^{\Omega} X_{\mu}\right)^{2}+\left[X_{\mu}, X_{\nu}\right]^{2}+2 V_{\mu \nu}^{\Omega}\left[X_{\mu}, X_{\nu}\right]\right\}
$$

The effective $S U(2)$ gluonic Lagrangian in variables $\left(V_{\mu}^{\Omega}, X_{\nu}\right)$ is

$$
\begin{gathered}
L=L_{a n}+L_{Y M}+\frac{\Lambda^{2}}{4 \pi^{2}} \operatorname{tr}\left(\partial_{\mu} \hat{n}\right)^{2}=L_{Y M}^{\Omega}+\frac{\Lambda^{2}}{4 \pi^{2}} \operatorname{tr}\left(\partial_{\mu} \hat{n}\right)^{2}+ \\
T+P+\left(\frac{1}{24 \pi^{2}}+\frac{1}{g^{2}}\right) \operatorname{tr}\left\{V_{\mu \nu}^{\Omega}\left[X_{\mu}, X_{\nu}\right]\right\}
\end{gathered}
$$


where $T$ is the kinetic term for $X_{\mu}$ and $(-P)$ is the potential

$$
\begin{gathered}
T=\left(\frac{1}{48 \pi^{2}}+\frac{1}{2 g^{2}}\right) \operatorname{tr}\left\{\left(D_{\mu}^{\Omega} X_{\nu}-D_{\nu}^{\Omega} X_{\mu}\right)^{2}\right\}+\frac{1}{24 \pi^{2}} \operatorname{tr}\left[D_{\mu}^{\Omega}, X_{\mu}\right]^{2} \\
P=\frac{\Lambda^{2}}{4 \pi^{2}} \operatorname{tr} X_{\mu}^{2}-\frac{1}{12 \pi^{2}} \operatorname{tr}\left(X_{\mu}^{2}\right)^{2}+\left(\frac{1}{24 \pi^{2}}+\frac{1}{2 g^{2}}\right) \operatorname{tr}\left[X_{\mu}, X_{\nu}\right]^{2}
\end{gathered}
$$

It follows that $\operatorname{tr} X_{\mu}^{2}$ can form a gauge invariant condensate $\left(\operatorname{tr} X_{\mu}^{2}\right)_{0}=-g^{2} \sigma / 2$ as a minimum of $-P$, and a mass appears. Denote a hermitian vacuum field by $\phi_{\mu}^{a}$, so that $\sigma=\left(\phi_{\mu}^{a} \phi_{\mu}^{a}\right)$ and $X_{\mu}=\left(\phi_{\mu}^{a}+Y_{\mu}^{a}\right) \tau_{a} / 2 i$. Then $\sigma=-9 \Lambda^{2} /\left(7 g^{2}+48 \pi^{2}\right)$ and $m_{Y}^{2}=-\frac{1}{3} \sigma b_{3}$, where $b_{3}=\left(g^{2}+12 \pi^{2}\right) / 24 \pi^{2} g^{2}$. The condensate $\sigma$ is negative, and the vacuum field $\phi_{\mu}^{a}$ is space-like.

The last term in $T$ should be analysed together with the gauge condition for $V_{\mu}$. In the flavor case, the term $(-P)$ without $\operatorname{tr}\left\{\left(X_{\mu}^{2}\right)^{2}\right.$ corresponds to the Skyrme Lagrangian. The lagrangian for the n-field [4] is contained in the first two terms of $L$.

The Lagrangian of [7] is $L_{Y M}\left(V^{\Omega}+X\right)$. New terms are contained in $L_{a n}$; they are partly built on structures already existing in $L_{Y M}$, but with different coefficients. Note, that an expression $\operatorname{tr}\left[D_{\mu}^{\Omega}, X_{\mu}\right]^{2}$ in color $L_{a n}$ looks as a standard gauge condition term, while in the flavor case $\left[D_{\mu} A_{\mu}\right]^{2}$ leads to ghosts. Last term in $T$ and first two terms in $P$ are quite new; they are specific for bosonization. To get more insight into meaning of $L_{a n}$ we need to assume a definite representation for $X_{\mu}$. Investigation of the effective Lagrangian is the next step of bosonization approach.

\section{Acknowledgments}

We are grateful to Dmitry Vassilevich for interesting discussions.

\section{References}

[1] S.L.Adler, Phys.Rev. 177, 2426 (1969); J.S.Bell and R.Jackiw, Nuovo Cim., A60, 47 (1969); W.A.Bardeen, Phys.Rev. 184, 1847(1969).

[2] Y.Nambu, Phys.Rev. D 10, 4262 (1974); G. 't Hooft, in: High Energy Physics, edited by A.Zichichi (Editotrice Compositori, Bologna, 1975); S.Mandelstam, Phys.Report, 23, 245 (1976): A.M.Polyakov, Nucl.Phys. B120,429 (1977).

[3] L. Faddeev and A. Niemi, Nature 387, 58 (1997).

[4] L.D.Faddeev, Quantization of Solitons, IAS preprint, IAS-75-QS70, Princeton, 1975

[5] R.Battye and P.Sutcliffe, Phys.Rev.Lett.81, 4798 (1998).

[6] S.Shabanov, Phys.Lett. B 458, 322 (1999); Phys.Lett. B 463, 263 (1999). 
[7] Y.M.Cho, Phys.Rev.D 21, 1080 (1980); Phys.Rev.D 23, 2415 (1981).

[8] Y.M Cho, Phys.Rev.Lett. 87, 252001 (2001), W.S.Bae, Y.M.Cho, and S.W.Kimm, Phys.Rev. D65, 025005. (2002); Y.M.Cho,H.W.Lee and D.G.Pak, Phys.Lett. B525, 347 (2002); hep-th/0201179.

[9] K.-I.Kondo, T.Murakani, T.Shinohara, hep-th/0504107; S.Kato et al. hep-ph/0509069.

[10] L.Faddeev annd A.Niemi, Phys.Rev.Lett. 85，3416 (2000); Phys.Lett.B525,195 (2002).

[11] A.Andrianov and Yu.Novozhilov, Phys.Lett.B 153, 422 (1985).

[12] V.Novozhilov and Yu.Novozhilov, Phys.Lett. B522, 49 (2001), hep-ph/0110006 Teor.Math.Phys. 131, 62 (2002).

[13] .Fujikawa, Phys.Rev.Lett.,42, 1195 (1979).

[14] A.A.Andrianov, V.A.Andrianov, V.Yu. Novozhilov and Yu.V.Novozhilov, Lett.Math.Phys. 11, 217 (1986).

[15] V.Yu.Novozhilov (in preparation). 